\title{
Giant Intraperitoneal Liposarcoma: About A Case and Review of the Literature
}

\author{
Fatiha Bellouhou $^{1 *}$, Fatima Ezzahra Haddar ${ }^{1}$, Sofia Oubaha ${ }^{2}$, Zouhour Samlani ${ }^{1}$, Khadija Krati ${ }^{1}$
}

${ }^{1}$ Department of Gastroenterology, Mohammed VI University Hospital, Marrakech, Morocco

${ }^{2}$ Department of Physiology, Faculty of Medicine and Pharmacy at Cadi Ayyad University, Marrakech, Morocco

DOI: $10.36347 /$ sjmcr.2020.v08i07.006

| Received: 27.01.2020 | Accepted: 03.02.2020 | Published: 18.07.2020

*Corresponding author: Fatiha Bellouhou

\section{Abstract}

Intraperitoneal liposarcoma is a very rare type of tumor, accounting for less than $1 \%$ of all malignancies. We report a case of 60-year-old man who was admitted with an enlarged abdomen, and computed tomography (CT) showed a, large hypoechoic lesion intraperitoneal measuring $37 \mathrm{~cm} / 28 \mathrm{~cm}$. The There is no occlusive syndrome, peritoneal effusion, or adenomegaly. The surgical procedure extracts a round fat mass whose cleavage planes are clean. The anatomopathological study shows lipoblasts with large nuclei and uncontrolled cell proliferation associated with vacuoles The patient did not receive any adjuvant therapy, and CT showed no evidence of recurrence during followup.

Keywords: Intraperitoneal liposarcoma, giant liposarcoma; surgical resection.

Copyright @ 2020: This is an open-access article distributed under the terms of the Creative Commons Attribution license which permits unrestricted use, distribution, and reproduction in any medium for non-commercial use (NonCommercial, or CC-BY-NC) provided the original author and source are credited.

\section{INTRODUCTION}

Liposarcoma is a very rare adipose-like malignant mesenchymal tumor. The world health organization classifies liposarcomas into five subtypes: well differentiated, myxoid, pleomorphic, dedifferentiated and mixed.

There are almost 50 histological types of soft tissue sarcoma and liposarcoma makes up to $9.8 \%$ to $18 \%$ of the soft tissue sarcoma family. Most of the patient present to clinicians as an incidental finding or the sheer size of the tumour causes compressive symptoms to the adjacent structure as the special feature of liposarcoma is its tendency to occur in visceral spaces,

In the light of a new observation of intraperitoneal liposarcoma and a review of the literature, we propose to analyze the epidemiological aspects, the diagnostic difficulties of this affection and to specify the therapeutic means and the prognostic elements.

\section{ObSERVATION}

We report a case of a 60 -year-old patient with no particular pathological history, who had progressive abdominal distension since 1 year associated with abdominal, peri-umbilical pain and chronic constipation with no associated digestive or extradigestive manifestations.

The patient is afebrile, with a deterioration of the general state made of a loss of $10 \mathrm{~kg}$ in 1 year and asthenia. The Palpation has detected an enormous renitente painless abdominal mass (Figure-1). Biological investigations reveal an inflammatory syndrome associated with iron deficiency anemia.

Abdominal ultrasound revealed a large hypoechoic lesion with posterior, limited, intraperitoneal reinforcement. This mass is renitent to the passage of the probe. An abdominopelvic CT is performed with injection of contrast medium.

The CT sections show a large mass of fat density, intra peritoneal (Figure-2) measuring $37 /$ 28/12 cm The digestive loops are pushed back without being invaded. There is no occlusive syndrome, peritoneal effusion, or adenomegaly.

The diagnosis of peritoneal liposarcoma is then proposed. The surgical procedure extracts a round fat mass whose cleavage planes are clean. The digestive loops are pushed back without trophic abnormality. 


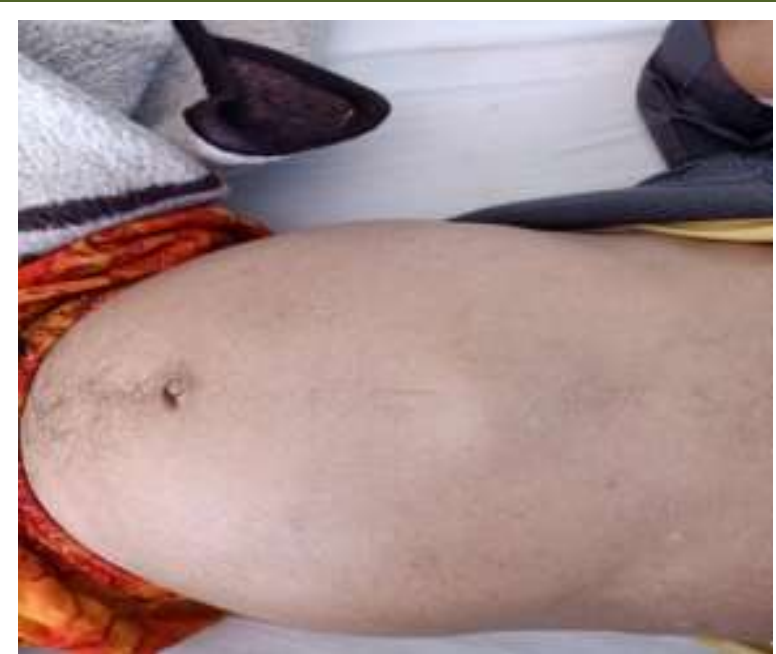

Fig-1: Clinical view of enormous abdominal mass

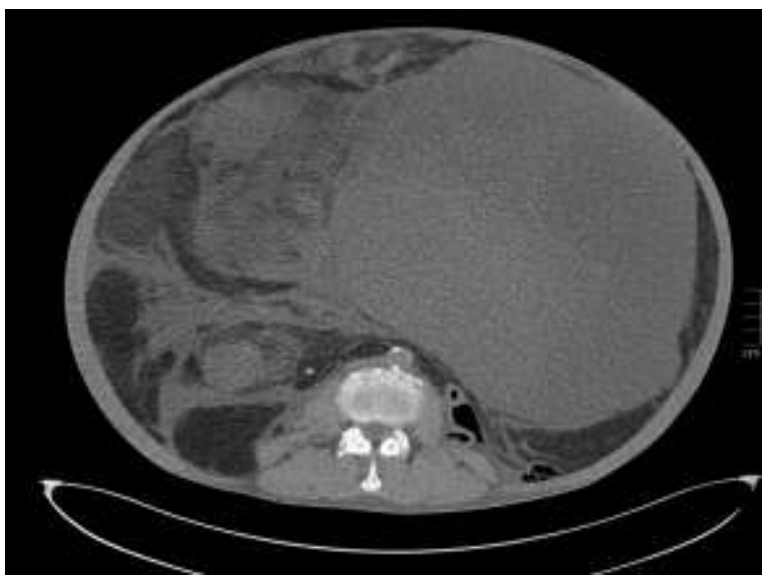

Fig-2: CT with intravenous contrast showing a mass displacing the adjacent bowel loops without evidence of obstruction

The anatomopathological study shows lipoblasts with large nuclei and uncontrolled cell proliferation associated with vacuoles (Figure-3). The postoperative course was regular and the patient was discharged on the 6th day. Our patient did not receive any adjuvant therapy, and CT showed no evidence of recurrence during follow-up.

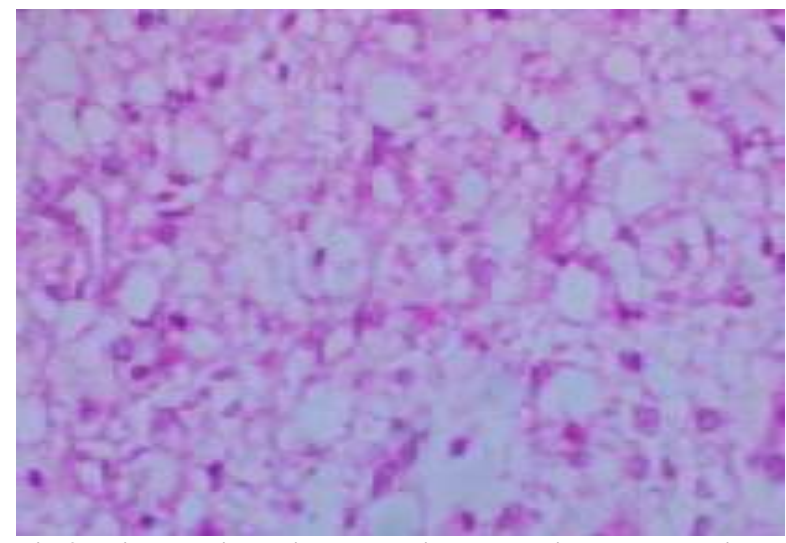

Fig-3: Microscopic section shows lipoblasts with large nuclei and uncontrolled cell proliferation associated with vacuoles

\section{DISCUSSION}

Peritoneal liposarcoma is a malignant tumor whose median survival does not exceed two years despite surgical excision [1]. Its discovery is late because it is often asymptomatic. Its frequency is estimated at $0.025 \%$ of operated digestive tumors [2]. Its tumor potential is often underestimated.

Liposarcoma mainly affects patients aged 50 to 70 years with male predominance, but without racial predominance. According to WHO liposarcoma can be classified into well-differentiated, myxoid, round-cell, pleomorphous and dedifferentiated liposarcoma [3].

Liposarcoma has a wide array of symptoms and signs.The growth rate of this tumor varies from case to case, usually this tumor has a very slow growth, and can remain quiescent for months, even years.

Clinically, patients complain of nonspecific abdominal pain and distention. They preserve for a long time a good general state.The mass can become too big and cause obstructive symptoms, which was the case of our patient who presented for abdominal pain evolving for 1 year associated with constipation and loss of weight and appetite [4]

Macroscopically, it is a voluminous mass well limited and regular, soft and yellowish. The tumor component is often necrotic. A Japanese team retrospectively correlated 10 peritoneal liposarcomas to the histological study and showed that the central tumor component is well differentiated (lipoblasts and sclerosis) in $50 \%$ of cases [5].

Radiologically, X-ray of the abdomen without preparation is not very contributive. Ultrasonography remains limited in topographic diagnosis and in the nature of the lesion. CT allows a positive diagnosis with density values in the Hounsfield scale [6]. MRI refines the nature of the tumor component by virtue of signal intensity characteristics: fluid T2 hypersignal for myxoid tumors, T2 hyposignal for sclerosant-type tumor [5].

The large surgical removal of the tumor carrying the neighboring organs, if necessary, is the only therapeutic alternative to these lesions. The interest of the adjuvant treatments is debatable. Radiotherapy used pre- or postoperatively seems to reduce the risk of recurrence. Given the low chemosensitivity, the benefit of chemotherapy is limited [7,8].

\section{CONCLUSION}

Peritoneal liposarcoma is a very rare adiposelike malignant mesenchymal tumor. A complete surgical resection with removal of the contiguous intra and retroperitoneal organs when infiltrated represents the only therapeutic option to obtain a negative margin 
and therefore an oncological radicality. More studies are needed to prove the efficiency of chemotherapy and adjuvant radiotherapy for local control and the increase of long-term survival.

\section{Conflict of interest: None.}

\section{REFERENCES}

1. Tsutsumi H, Ohwada S, Takeyoshi I, Izumi M, Ogawa T, Fukusato T, Morishita Y. Primary omental liposarcoma presenting with torsion: a case report. Hepato-gastroenterology. 1999;46(27):2110-2.

2. Kurosaki Y, Tanaka YO, Itai Y. Welldifferentiated liposarcoma of the retroperitoneum with a fat-fluid level: US, CT, and MR appearance. European radiology. 1998 Mar $1 ; 8(3): 474-5$.
3. WHO. Classification of Tumours of soft Tissue and Bone. 2002; 35-46.

4. Pack GT, Pierson JC. Liposarcoma: a study of 105 cases. Surgery. 1954 Oct 1;36(4):687-712.

5. Kim T, Murakami T, Oi H, Tsuda K, Matsushita M, Tomoda K, Fukuda H, Nakamura H. CT and MR imaging of abdominal liposarcoma. AJR. American journal of roentgenology. 1996 Apr;166(4):829-33.

6. Dupraz B, Hauser H, Jacot-Des-Combes E. Contribution de la tomodensitométrie dans le diagnostic de liposarcome. J Belge Radiol. 1991;74:269-75.

7. Bennani S, Debbagh A, Louahlia S, El Mrini M, Benjelloun $\mathrm{S}$. Le liposarcome rétropéritonéal: à propos de deux cas. InAnnales d'urologie 1995 ; 29(3), 154-158.

8. Dei Tos AP. Liposarcoma: new entities and evolving concepts. Annals of diagnostic pathology. 2000 Aug 1;4(4):252-66. 